\title{
Reliability analysis of stochastic park-and-ride network
}

\author{
Wenbo FAN ${ }^{*}$ \\ School of Transportation and Logistics, Southwest Jiaotong University, Chengdu 610031, China
}

\begin{abstract}
Park-and-ride (P\&R) facilities can alleviate the traffic burden in central urban areas by enabling car drivers to park at the perimeter of congested areas and continue their journeys with public transportation (e.g., metro and bus rapid transit). Whether a P\&R scheme is successful depends on its attractiveness to car users. This paper presents an evaluation method for the reliability analysis of $P \& R$ mode. Two indices, $P \& R$ reliability and mode reliability, are introduced to represent the reliabilities of a transfer point and an entire trip, respectively. Then, a systematic reliability analysis is conducted for a stochastic P\&R network, where travelers can complete their journeys via two options: auto mode or P\&R mode. A variational inequality (VI) model is proposed and solved by a heuristic solution algorithm. Numerical results show that the P\&R facility reliability is significantly influenced by the capacity of parking facilities, the dispatching frequency of the connecting metro, and the metro fare. In addition, a higher level of total demand in the network has significant negative impacts on P\&R mode's attractiveness compared to auto mode.
\end{abstract}

Key words: park-and-ride facility; stochastic user equilibrium; reliability; variational inequality model

(C) 2012 JMT. All rights reserved.

\section{Introduction}

$\mathrm{P}$ ark-and-ride (P\&R) facilities have been used by commuters for several decades, and were initially designed to attract public transit patrons. Nowadays, increasing number of urban policy-makers recognize that the $P \& R$ mode is an effective approach to mitigating both road congestion and environmental problems, because it possesses the advantages of both the auto and public transportation modes. The P\&R mode of transportation allows car drivers to park at transfer sites and embark on public transportation systems to complete the remaining portion of their trips. In under-populated areas, transportation by car is usually the favored mode of transportation due to lack of public transportation systems and lack of congestion. Then, in congested urban areas, efficient and safe public transportation allows commuters to save time by avoiding having to deal with traffic congestion and limited parking space.

In reviews of the effectiveness of $P \& R$ facilities worldwide, studies have found that there are both successes (the Oxford's $P \& R$ ) and failures (the Amsterdam's $P \& R$ ) [1-2]. In stances of failures are in part due to poor predictions of the prospective user population.

To attract users, P\&R facilities must be accessible, of high quality in terms of infrastructure and they must

Received Feb. 13, 2012; revision accepted Mar. 10, 2012

*Corresponding author. E-mail: dr.fanwenbo@qq.com

(C) 2012 JMT. All rights reserved

doi: 10.3969/j.issn.2095-087X.2012.01.009 have an efficient and reliable connection to a public transportation system $[1,3]$. Among these criteria, the reliability of the connecting public transportation systems has a great impact on car drivers' choice and accordingly on the network performance $[4,5]$. Commuters expect stable and reliable transportation service, especially in our modern world where people are more conscious of the value of time.

In general, travel uncertainty is related to a group of factors, including travel time, connectivity of transportation networks, level of service (LOS), etc. In order to measure how this travel uncertainty affects travelers' decisions, the concept of transport reliability was introduced. Transport reliability is typically defined as the probability that a transportation system accomplishes its expected goals or functions according to certain constraints (e.g., travel time). Previous studies have considered the reliability of road network, transit service, and parking facilities [6-8]. However, these studies do not examine the effectiveness of the P\&R network with respect to the uncertainty associated with parking and waiting for connecting public transportation.

The purpose of this study is to assess the reliability of a stochastic P\&R network, and to report analytical results with respect to both the reliability and the overall network performance. In particular, we consider how reliability and network performance are affected by the capacity of the parking facility, the public transportation fee, the dispatching frequency, and the total demand. 


\section{Supply function}

Consider a P\&R network $G=(N, L)$, where $N$ is the set of nodes, including origins, destinations and intermediate nodes of all trips; and $L$ is the set of links connecting nodes. The network $G$ is composed of the auto subnetwork $G_{\mathrm{a}}=\left(N_{\mathrm{a}}, L_{\mathrm{a}}\right)$, the metro sub-network $G_{\mathrm{b}}=\left(N_{\mathrm{b}}, L_{\mathrm{b}}\right)$, the set of parking lots, the set of P\&R sites, and the links which describe the walking paths. Let $R$ and $S$ be the sets of origins and destinations of possible trips, respectively. Then we have $r \in R \subset N$ and $s \in S \subset N$, where $r$ and $s$ denote a particular origin and destination. Let $I_{r s}$ and $T_{r s}$ denote the sets of all feasible parking lots and P\&R sites between origin-destination (OD) pair $(r, s), i \in I_{r s}, t \in T_{r s}$. Last, let $m$ denote the set of travel modes, and $m=(x, y)$ represents auto mode and $\mathrm{P} \& \mathrm{R}$ mode.

\subsection{Auto travel time on roads}

In reality, there are a variety of irregular and random events, which occur on the auto journey, such as vehicle collisions, vehicle breakdowns, signal failures, and adverse weather events. Thus, it is assumed in this study that the link travel time $c_{l}$ on the auto sub-network is a stochastic variable which follows an independent normal distribution, i.e.,

$$
c_{l} \sim N\left(\tau_{l},\left(\rho_{l} t_{l}\right)^{2}\right),
$$

where $\rho_{l}$ reflects the stochasticity of the auto subnetwork [9]. Let $T_{r j, p}$ be the travel time from origin $i$ to node $j$ (either destination $s$ or parking facility $t$ ) via route $p$, which can be described by

$$
T_{r j, p}=\sum_{l \in L_{\mathrm{a}}} c_{l}\left(v_{l}\right) \delta_{l p}^{r j},
$$

where $\delta_{l p}^{r j}$ is an indicator function that equals 1 if link $l$ belongs to path $p$, and 0 otherwise, and $v_{l}$ is the traffic flow on link $l$. From the additive property of normal random variables, we know that $T_{r j, p}$ also follows a normal distribution with expectation and standard deviation respectively given by

$$
\begin{gathered}
E\left[T_{r j, p}\right]=\sum_{l \in L_{a}} \tau_{l}\left(v_{l}\right) \delta_{l p}^{r j}, \\
\sigma_{r j, p}=\sqrt{\sum_{l \in L_{a}}\left(\rho_{l} t_{l}\right)^{2} \delta_{l p}^{r j}},
\end{gathered}
$$

where $\tau_{l}$ is a function which describes the volume of the flow on link $l$. We write $\tau_{l}$ in the form recommended by BPR (Bureau of Public Roads):

$$
\tau_{l}\left(v_{l}\right)=\tau_{l}^{0}\left(1+0.15\left(v_{l} / C_{l}\right)^{4}\right),
$$

where $\tau_{l}^{0}$ and $C_{l}$ are the free-flow travel time and the capacity on link $l$, respectively. The link flow $v_{l}$ has the following relationship with path flow $f_{r j, p}$ :

$$
v_{l}=\frac{1}{\gamma} \sum_{(r, j)} \sum_{p \in P_{r j}} f_{r j, p} \delta_{l p}^{r j},
$$

where parameter $\gamma$ converts the path flow from passenger units into vehicular units.

\subsection{In-vehicle time by metro}

For the metro sub-network, the time in vehicle can be treated as a deterministic variable due to the exclusive tracks. In addition, to consider congestion within a vehicle, Li et al. [5] introduced a BPR-form function:

$$
b_{l}\left(v_{l}^{b}\right)=b_{l}^{0}\left(1+0.1\left(\frac{v_{l}^{b}}{C_{l}^{b}}\right)^{2.0}\right),
$$

where $b_{l}, v_{l}^{b}$, and $C_{l}^{b}$ are, respectively, time spent in vehicle, the vehicle passenger volume and the capacity on metro link $l$. Then let $T_{r s, p}^{b}$ be time spent in vehicle on metro path $p$, which is given by

$$
T_{r s, p}^{b}=\sum_{l \in L_{b}} b_{l}\left(v_{l}^{b}\right) \delta_{l p}^{r s}
$$

\subsection{Parking search time}

Within parking lots at the final destination or at intermediate transfer points, commuters spend time searching for an available space. Following Ref. [5], one knows that the parking search time $\zeta_{j}$ is also normally distributed with a mean $d_{j}$ calculated by

$$
d_{j}\left(v_{j}\right)=d_{j}^{0}+0.31\left(\frac{v_{j}}{C_{j}}\right)^{4.03}
$$

where $d_{j}^{0}, v_{j}$, and $C_{j}$ are the free-flow parking search time, the volume of parking flow, and the capacity of the parking facility $j$, respectively.

\subsection{Waiting time at metro station}

Let $T_{t}^{w}$ be the waiting time at the station which connects the P\&R at site $t$ to the public transportation system. This waiting time is related to the dispatching frequency $\left(F^{b}\right)$ of the metro and the distribution of pas- 
senger arrival times. Suppose that the time at which passengers arrive at the station follows a uniform distribution [10]. Then we have $T_{t}^{w} \sim\left(0,1 / F^{b}\right)$ with mean and standard deviation as below:

$$
\begin{aligned}
& E\left[T_{t}^{w}\right]=1 / 2 F^{b}, \\
& \sigma_{t}=1 / 2 F^{b} \sqrt{3} .
\end{aligned}
$$

\section{Demand function}

Because of the uncertainty of the P\&R network, commuters incur costs that may be a result of either arriving late, or arriving early to ensure that the destination is reached by the required time, as well as the ordinary costs such as auto travel time/in-vehicle time, parking search time, waiting time, and parking fees. To include the effects of these unreliability-related costs into travelers' choices, Noland et al. [11] explicitly formulated the travel disutility function:

$$
U_{r s, p}^{m}=E\left[\varphi_{r s, p}^{m}\right]+\theta \sigma_{r s, p}^{m},
$$

where $\varphi_{r s, p}^{m}$ denotes a generalized cost that is often formulated as a weighted sum of these ordinary time and fee cost incurred [12]; $\theta$ and $\sigma_{r s, p}^{m}$ reflect the user's value of reliability [13] and the additional costs incurred from an unreliable journey. These additional costs denoted by $\sigma_{r s, p}^{m}$ are calculated by

$$
\sigma_{r s, p}^{m}=\sqrt{\sum_{l \in L_{\mathrm{ra}}}\left(\delta_{l p} \sigma_{l}^{a}\right)^{2}+\left(\delta_{j p} \sigma_{j}^{m}\right)^{2}+\left(\delta_{t p} \sigma_{t}^{b}\right)^{2}},
$$

where $\sigma_{l}^{a}, \sigma_{j}^{m}$, and $\sigma_{t}^{b}$ are the standard deviations of the auto travel time, the parking search time, and the waiting time, respectively.

Then, we determine a traveler's perceived disutility:

$$
\hat{U}_{r s, p}^{m}=U_{r s, p}^{m}+\alpha\left(\xi_{r j, p}^{a}+\delta_{j p} \varepsilon_{j}^{m}+\delta_{t p} \varepsilon_{t}^{b}\right),
$$

where $\xi_{r j, p}^{a}, \varepsilon_{j}^{m}$, and $\varepsilon_{t}^{b}$ are the traveler's perceived error of the expected auto travel time, parking search time, and waiting time, respectively, and follow the normal distribution [5]. The value of time $\alpha$ converts the perception errors from time units into disutility units.

In this study, we assume that travelers make their decisions in order to minimize their perceived disutility. Therefore, the probability that an alternative travel time or mode is chosen given by

$$
P_{r s, p}^{m}=P\left\{\hat{U}_{r s, p}^{m}<\hat{U}_{r s, q}^{h} \mid \forall h \neq m, q \neq p\right\},
$$

where $P_{r s, p}^{m}$ is the probability of choosing between $r$ and $s$ by mode $m$ via path $p$. This probability of choosing an alternative travel route or mode depends on the distribution of the perception errors.

Finally, the path flow can be obtained by

$$
f_{r s, p}^{m}=q_{r s} P_{r s, p}^{m},
$$

where $q_{r s}$ is the total travel demand between $r$ and $s$.

\section{Stochastic user equilibrium and a solu- tion algorithm}

Considering the asymmetric effects of flows of two modes on link travel time, we present a variational inequality (VI) formulation which is equivalent to the stochastic user equilibrium conditions of Eqs. (11) to (15). These equilibrium conditions can be stated as

$$
\sum_{r s} \sum_{m} \sum_{p \in P_{r s}} G_{r s, p}^{m}\left(f_{r s, p}^{m}-f_{r s, p}^{m^{*}}\right) \geq 0
$$

where, according to Ref. [14],

$$
G_{r s, p}^{m}=\left(f_{r s, p}^{m}-q_{r s} P_{r s, p}^{m}\right) \frac{\partial U_{r s, p}^{m}}{\partial f_{r s, p}^{m}} .
$$

The feasible route flows can be obtained using nonnegativity constraints and conservation conditions:

$$
\Omega=\left\{f \mid f_{r s, p}^{m} \geq 0, \sum_{r s} \sum_{m} \sum_{p} f_{r s, p}^{m}=q_{r s}\right\} .
$$

Note that if all functions in equation (16) are continuous with regard to route flows, then there exists at least one solution to the proposed VI model according to Brouwer's fixed-point theorem [15]. To solve such a stochastic user equilibrium problem, the method of successive averages (MSA) is often adopted in combination with the Monte Carlo (MC) method which simulates the uncertainty of the network and the personal perception. The algorithm is implemented as follows:

Step 1 Initialize both the counter $n=1$ and the set of route flows $f^{(n)}$.

Step $2 \mathrm{MC}$ sampling. Set the sampling variable to $\kappa=1$.

Step 3 Stochastic network loading.

Step 3.1 Calculate the link flows on the P\&R networks $v_{l}^{m(\kappa)}$, the auto travel times $\tau_{l}^{(\kappa)}$, the in-vehicle times $b_{l}^{(\kappa)}$ and the parking search times $d_{j}^{(\kappa)}$.

Step 3.2 Us MC to sample ramdom errors and calculate perceived disutilities $\hat{U}_{r s, p}^{m(\kappa)}$. 
Step 3.3 Load the travel demand variable $q_{r s}$ to the travel alternatives function with the minimal perceived disutility to get the auxiliary route flows $\tilde{g}^{(\kappa)}$.

Step 3.4 In the inner loop, update route flows:

$$
g^{(\kappa)}=\left[(\kappa-1) g^{(\kappa-1)}+\tilde{g}^{(\kappa)}\right] / \kappa .
$$

Step 3.5 If the sampling variable $\kappa$ reaches a prespecified sample size, then stop the inner loop and assign $g^{(n)}=g^{(\kappa)}$; otherwise set $\kappa=\kappa+1$ and go to Step 3 .

Step 4 In the outer loop, update route flows:

$$
f^{(n+1)}=f^{(n)}+\left(g^{(n)}-f^{(n)}\right) / n .
$$

Step 5 Check convergence by checking if

$$
G=\left\|f^{(n+1)}-f^{(n)}\right\| /\left\|f^{(n)}\right\|<\varepsilon,
$$

where $\varepsilon$ is a pre-specified precision: if the inequality holds, then stop; otherwise, let $n=n+1$ and go to Step 2 .

\section{Reliability analysis}

\subsection{Definition}

Reliability analysis has received substantial attention in fields which study transportation [8]. This paper focuses on the P\&R network, and introduces two new indices to describe the quality of the reliability of the P\&R facility and the P\&R mode, respectively, which are defined as follows.

Definition 1 P\&R (facility) reliability is the probability of completing a transfer within a certain time threshold (i.e., a pre-given $k_{1}$ ) at a P\&R site, and is given by

$$
\begin{aligned}
& A_{t}\left(\zeta_{t}+w_{t}+T_{t}^{w} \leq k_{1}\right)= \\
& \quad \iint_{\zeta_{t}+T_{t}^{w} \leq k_{1}-w_{t}} \hbar\left(\zeta_{t}, T_{t}^{w}\right) \mathrm{d} \zeta_{t} \mathrm{~d} T_{t}^{w},
\end{aligned}
$$

where $w_{t}$ is the walking time from the parking facility to the metro station at the P\&R site and treated as a deterministic variable; and $\hbar\left(\zeta_{t}, T_{t}^{w}\right)$ is the joint probability density function of stochastic parking search time $\zeta_{t}$ and waiting time $T_{t}^{w}$ and is given by the function:

$$
\hbar\left(\zeta_{t}, T_{t}^{w}\right)=\left\{\begin{array}{c}
\frac{F_{b}}{\sqrt{2 \pi} \sigma_{t}} \exp \left(\frac{\left(\zeta_{t}-d_{t}\right)^{2}}{-2 \sigma_{t}^{2}}\right), \quad 0<T_{t}^{w}<\frac{1}{F_{b}}, \\
0, \quad \text { otherwise. }
\end{array}\right.
$$

Thus, the P\&R reliability can be formulated as

$$
\begin{aligned}
& A_{t}\left(k_{1}\right)=\int_{k_{1}-\frac{1}{F_{b}}-w_{t}}^{k_{1}-w_{t}} \frac{F_{b}\left(k_{1}-\zeta_{t}-w_{t}\right)}{\sqrt{2 \pi} \sigma_{t}} \exp \left(\frac{\left(\zeta_{t}-d_{t}\right)^{2}}{-2 \sigma_{t}^{2}}\right) \mathrm{d} \zeta_{t} \\
& \quad+\int_{-\infty}^{k_{1}-\frac{1}{F_{b}}-w_{t}} \frac{1}{\sqrt{2 \pi} \sigma_{t}} \exp \left(\frac{\left(\zeta_{t}-d_{t}\right)^{2}}{-2 \sigma_{t}^{2}}\right) \mathrm{d} \zeta_{t} .
\end{aligned}
$$

The indicator reflects the LOS of a P\&R facility in a uncertain environments, especially when there is a lack of parking spaces or when the trains arrive infrequently at the metro station.

Definition 2 Mode reliability is the average probability that all commuters complete their entire journeys using a certain mode within a certain time threshold (i.e., $k_{2}$ ). This index can help reveal the effects of unreliability on a commuter's choice of mode and is formulated as

$$
A_{r s, p}^{m}\left(k_{2}\right)=\frac{\sum_{p \in P_{r s}} A_{r s, p}^{m}\left(\mu_{r s, p}^{m}<k_{2}\right) f_{r s, p}^{m}}{\sum_{p \in P_{r j}} f_{r s, p}^{m}},
$$

where $\mu_{r s, p}^{m}$ is the total journey time using mode $m$.

According to the additive property of normally distributed random variables, auto journey time $\mu_{r s, p}^{x}$ is also normally distributed. The reliability of the auto mode is found to be

$$
A_{r s, p}^{x}\left(k_{2}\right)=\Phi\left(\frac{k_{2}-\left(\sum_{l \in L_{\mathrm{m}}} \tau_{l}^{x} \delta_{l p}+d_{i}+w_{x}\right)}{\sqrt{\sum_{l \in L_{a}}\left(\sigma_{l}^{x}\right)^{2} \delta_{l p}+\left(\sigma_{i}\right)^{2}}}\right),
$$

where $\Phi(\cdot)$ is the cumulative distribution function the normal distribution, and $w_{x}$ is the total walking time taken when using auto mode.

The derivation for the reliability of the $P \& R$ mode is similar to the derivation of the P\&R reliability. The final formulation reliability of the P\&R mode is

$$
\begin{gathered}
A_{r s, p}^{y}\left(\mu_{r s, p}^{y} \leq k_{2}\right)=\int_{k_{2}-\frac{1}{F_{b}}}^{k_{2}} \frac{F_{b}\left(k_{2}-\Gamma_{r s, p}^{y}\right)}{\sqrt{2 \pi} \sigma\left(\Gamma_{r s, p}^{y}\right)} \exp \left(\frac{\left(\Gamma_{r s, p}^{y}-E\left(\Gamma_{r s, p}^{y}\right)\right)^{2}}{-2 \sigma\left(\Gamma_{r s, p}^{y}\right)^{2}}\right) \mathrm{d} \Gamma_{r s, p}^{y}+ \\
\int_{-\infty}^{k_{2}-\frac{1}{F_{b}}} \frac{1}{\sqrt{2 \pi} \sigma\left(\Gamma_{r s, p}^{y}\right)} \exp \left(\frac{\left(\Gamma_{r s, p}^{y}-E\left(\Gamma_{r s, p}^{y}\right)\right)^{2}}{-2 \sigma\left(\Gamma_{r s, p}^{y}\right)^{2}}\right) \mathrm{d} \Gamma_{r s, p}^{y},
\end{gathered}
$$


where $\Gamma_{r s, p}^{y}$ is the total journey time when using the P\&R mode but does not include the time spent waiting at the metro station. The total journey time also follows a normal distribution with expectation and standard deviation given by

$$
\begin{aligned}
& E\left(\Gamma_{r s, p}^{y}\right)=\left(\sum_{l \in L_{\mathrm{a}}} \tau_{l}^{x} \delta_{l p}+d_{t}+\sum_{l \in L_{b}} b_{l} \delta_{l p}+w_{y}\right), \\
& \sigma\left(\Gamma_{r s, p}^{y}\right)=\sqrt{\sum_{l \in L_{\mathrm{a}}}\left(\sigma_{l}^{x}\right)^{2} \delta_{l p}+\sigma_{t}^{2}} .
\end{aligned}
$$

\subsection{Numerical data input}

To illustrate the application of the proposed reliability analysis framework, we use our approach to analyze the reliability of the P\&R network shown in Fig. 1. There is one OD pair (1-2) and eight nodes in total. Node 1 lies in a suburban residential area; node 2 in the central business district (CBD); nodes $A$ and $B$ are two parking lots within the CBD; and P\&R sites 1 and 2 are located near to nodes 7 and 4, respectively. All nodes are connected by links (auto/metro/walk) that are described according to the parameters given in Table 1.

We assume that the length of metro link $(7,8)$ is $36 \mathrm{~km}$; the capacity and dispatching frequency are 400 passengers per car and 6 cars per hour, respectively; the average speed of metro train is $60 \mathrm{~km} / \mathrm{h}$; and the metro fare is CNY 0.4/km. Parking lots A and B have a capacity of 600 and $850 \mathrm{pcu}$ with free-flow parking search time of $0.1 \mathrm{~h}$, while $P \& R$ sites 1 and 2 have capacities of $400 \mathrm{pcu}, 600 \mathrm{pcu}$ and a free-flow parking search time

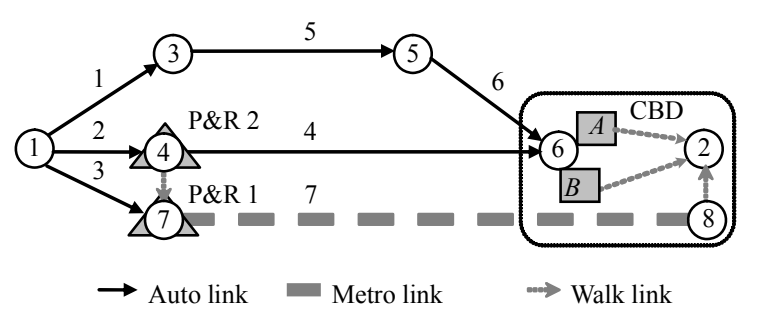

Fig. 1 A P\&R network example

Table 1 Parameters of the link travel time functions

\begin{tabular}{ccccc}
\hline Link & $\tau_{l 0}(\mathrm{~h})$ & $\begin{array}{c}C_{l} \\
\left(\mathrm{pcu} \cdot \mathrm{h}^{-1}\right)\end{array}$ & $\begin{array}{c}\text { Walking } \\
\text { link }\end{array}$ & $\begin{array}{c}\text { Walking } \\
\text { time }(\mathrm{h})\end{array}$ \\
\hline 1 & 0.30 & 800 & $(4,7)$ & 0.10 \\
2 & 0.15 & 800 & $(8,2)$ & 0.20 \\
3 & 0.20 & 800 & $(A, 2)$ & 0.10 \\
4 & 0.85 & 800 & $(B, 2)$ & 0.20 \\
5 & 0.55 & 800 & & \\
6 & 0.15 & 800 & & \\
\hline
\end{tabular}

of $0.05 \mathrm{~h}$. The parking fees at the parking lots and $\mathrm{P} \& \mathrm{R}$ sites are CNY 12 and 2, respectively. Other parameters include: total demand $q_{12}=2000 \times 1.0$ passenger per hour, value of reliability $\theta=2.0$, value of time $\alpha=1.0$, pre-specified time thresholds $\left(k_{1}, k_{2}\right)=(0.4,1.2)$, and $(0.5,0.3)$ reflect the stochasticity of the auto links and the parking facilities. Lastly, the MC sample size is $\kappa=2000$, unless the convergence is reached.

\subsection{Numerical analysis}

Table 2 shows the impacts of an unreliable network on mode splits. It shows that models that do not consider the effects of reliability may underestimate P\&R mode's share rate, as some of auto travelers would change travel plans to ensure a more reliable journey.

Tables 3-4 and Figs. 2(a) and 3(a) illustrate the effects of the dispatching frequency and the metro fare on the share of $P \& R$ mode, respectively. It can be seen that the share of $P \& R$ mode increases with either the increase of dispatching frequency or the decrease of metro fare. The reliability of the P\&R network is more sensitive to the metro fare than to the dispatching frequency. We hypothesize that waiting at the metro station mean more reliable travel due to the rising frequency of train arrivals, the reliability of P\&R sites may negate this effect because of higher P\&R demand.

Figs. 2(b) and 3(b) reveal flow splits which resulted from changes in the dispatching frequency and metro fare. Fig. 2(b) shows that P\&R 2 which has a higher capacity attracts more users due to its more reliable service. In Fig. 3(b), the share of P\&R 1 rises as metro fare increases. The explanation for this could be that a higher fare dissuades many $P \& R$ users, which in turn increases the reliability at $\mathrm{P} \& \mathrm{R}$ sites. As a result, some

Table 2 Impacts of unreliability on mode splits

\begin{tabular}{cccc}
\hline Sharing rate & Mode $x$ & Mode $y$ & OD \\
\hline Without UCs $^{*}$ & 54.18 & 45.82 & 100 \\
With UCs & 46.25 & 53.75 & 100 \\
UCs (CNY/person) & 1.08 & 0.46 & 0.78 \\
\hline
\end{tabular}

*UCs are the additional costs caused by unreliability.

Table 3 Effects of dispatching frequency on P\&R mode

\begin{tabular}{ccccc}
\hline \multirow{2}{*}{ Frequency } & \multirow{2}{*}{ Share (\%) } & \multicolumn{3}{c}{ Reliability (\%) } \\
\cline { 3 - 5 } & & Site 1 & Site 2 & Mode \\
\hline 2 & 47.28 & 29.72 & 31.90 & 34.60 \\
4 & 52.60 & 30.76 & 32.30 & 45.21 \\
6 & 53.75 & 34.96 & 35.93 & 50.94 \\
8 & 54.44 & 36.04 & 38.29 & 52.91 \\
\hline
\end{tabular}


Table 4 Effects of metro fare on P\&R mode

\begin{tabular}{ccccc}
\hline \multirow{2}{*}{$\begin{array}{c}\text { Fare } \\
(\mathrm{CNY} / \mathrm{km})\end{array}$} & Share (\%) & \multicolumn{3}{c}{ Reliability (\%) } \\
\cline { 3 - 5 } & & Site 1 & Site 2 & Mode \\
\hline 0.8 & 32.32 & 99.98 & 99.99 & 99.22 \\
0.6 & 43.42 & 91.78 & 97.88 & 93.00 \\
0.4 & 53.73 & 34.96 & 35.93 & 50.94 \\
0.2 & 61.83 & 7.70 & 6.26 & 13.93 \\
\hline
\end{tabular}

of the remaining P\&R users would switch to $P \& R 1$ because it is closer to the metro station.

Fig. 4 demonstrates the impact of changes in the total demand on the P\&R networks. The results show that increasing network demand reduces the reliability and the share of the P\&R mode, while the share of the auto mode increases. This could be because increased demand increases congestion on the metro sub-network which would lead to an increase in in-vehicle times. As

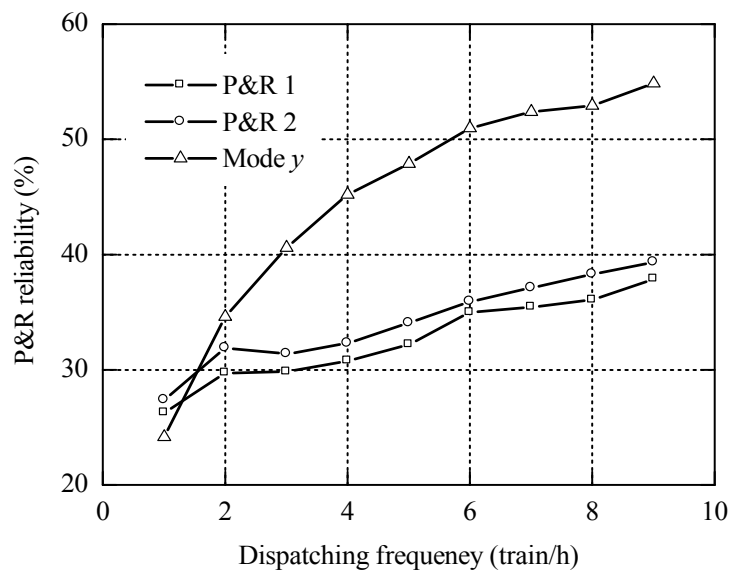

(a) Changes of the reliability a consequence of this increased congestion, some P\&R users may switch back to using auto mode.

Fig. 5 shows the effects of changing the prespecified time thresholds. It is shown in Fig. 5(a) that the P\&R reliability reaches $99 \%$ with $k_{1} \geq 0.7 \mathrm{~h}$. However, this means that commuters have to spend more time traveling as a result of either departing earlier or delaying activities to assure such high reliability. In reality, commuters chose to balance between incurred costs and a reliable journey. Li and Huang [16] studied these balancing behaviors by introducing schedule times into traveler's disutility function, which is beyond the scope of this paper.

Fig. 5(b) indicates that the P\&R mode reliability is more sensitive to the change of $k_{2}$. This can be explained by the lower stochasticity of P\&R mode, which means that is more less likely to complete the entire journey in a short time but more likely to complete that same journey in a longer time.

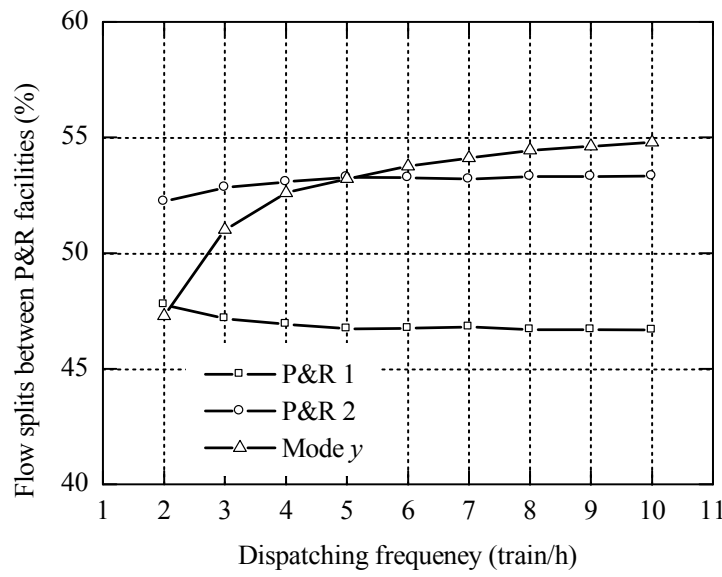

(b) Changes of flow splits

Fig. 2 Effects of dispatching frequency on P\&R mode

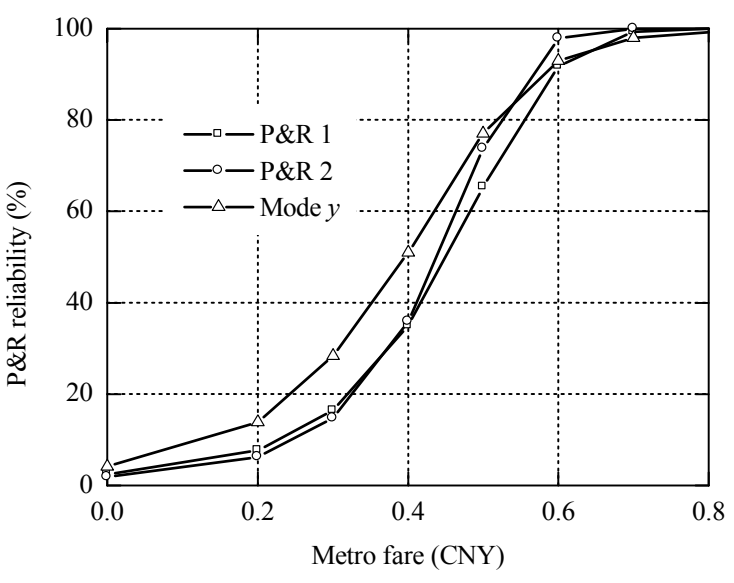

(a) Changes of the reliability

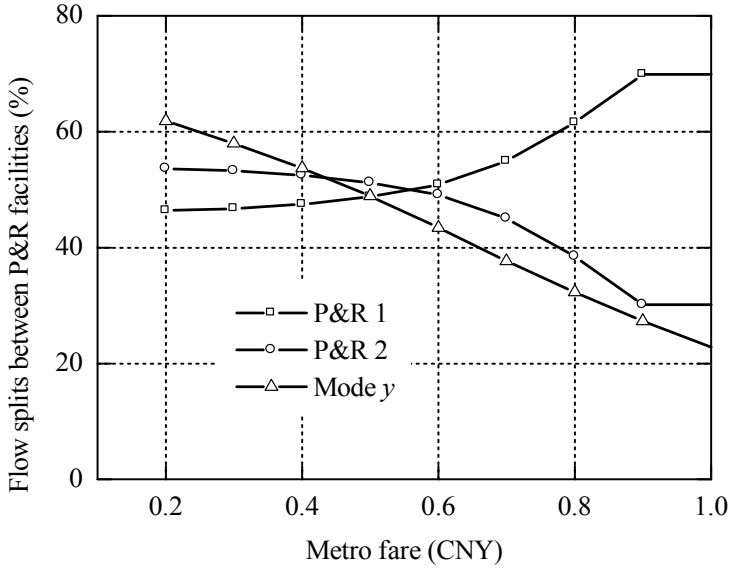

(b) Changes of flow splits

Fig. 3 Effects of metro fare on P\&R mode 


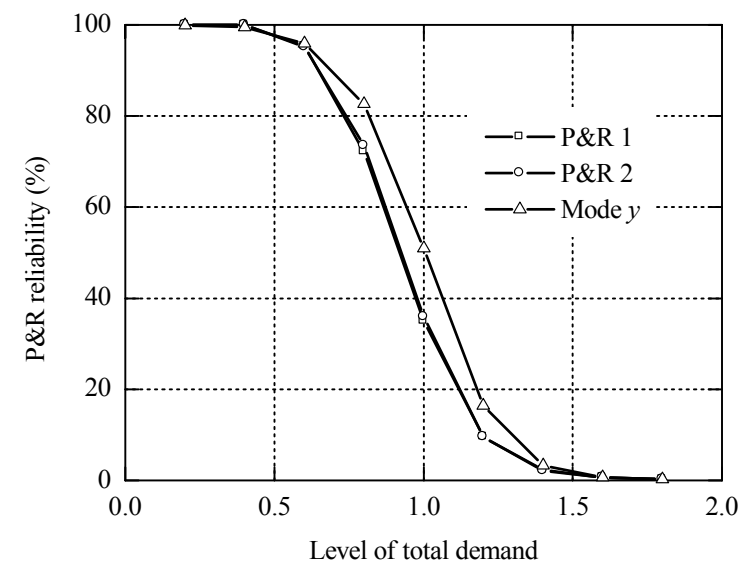

(a) Changes of the reliability

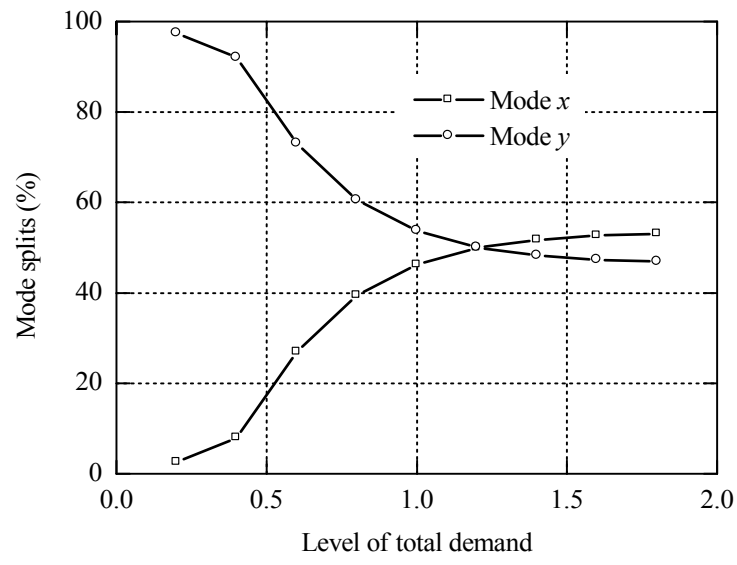

(b) Changes of mode splits

Fig. 4 Effects of the total travel demand on P\&R mode

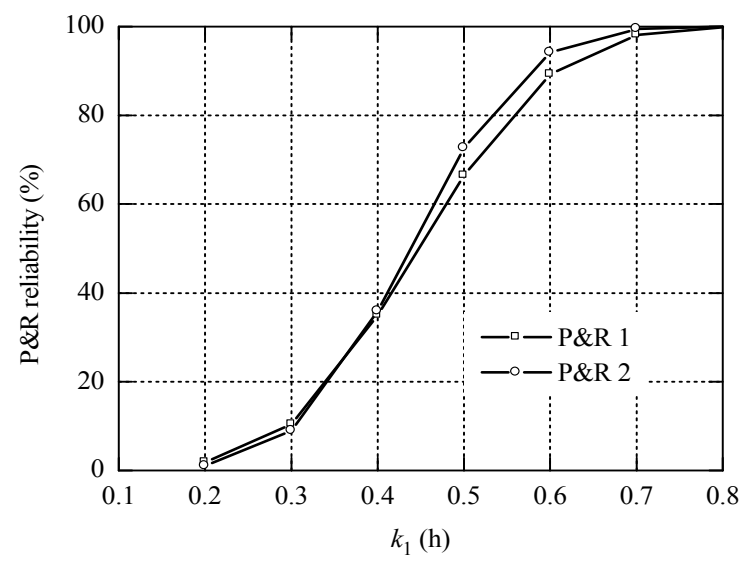

(a) Changes of $P \& R$ reliability

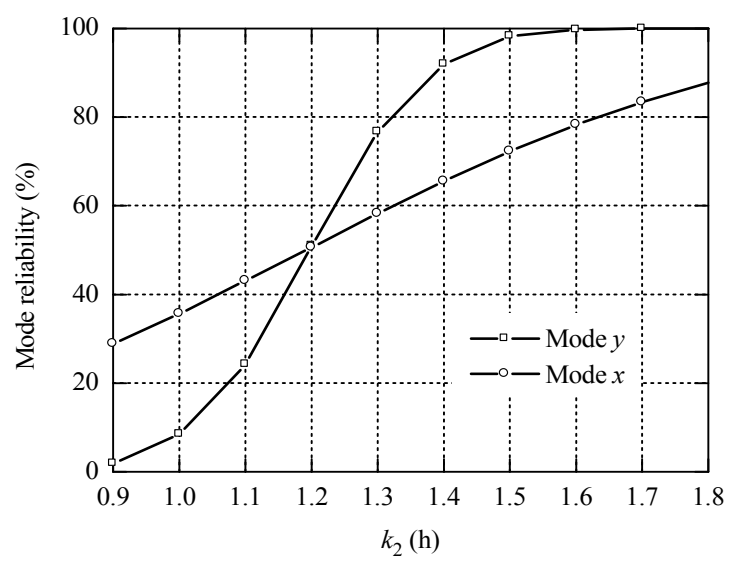

(b) Changes of mode reliability

Fig. 5 Model reliability with different pre-specified time thresholds

\section{Conclusion}

This paper proposes new indices of P\&R reliability and mode reliability to evaluate the LOS of a stochastic P\&R network. Additional costs caused by unreliability, which do not include the ordinary costs (e.g., travel time and fees), are introduced to the traveler's choice model. The stochastic user equilibrium is formulated as a VI problem. A heuristic algorithm to find the solution is designed.

To illustrate the applicability of these proposed models and algorithm, numerical simulations are presented. Firstly, the effects of unreliability on network performance are examined. We show that traditional models with only ordinary costs may underestimate the demand of P\&R facilities. Secondly, the study assesses the sensitivity of reliability with respect to several attributes, such as the dispatching frequency, the metro fare, the total demand, and the pre-given time thresholds. Results show that P\&R mode is less attractive than auto mode when the total travel demand is higher. This result suggests that some Chinese major cities with high population densities may expect a less effective P\&R system than those developed in European cities due to increase demand. Despite this, P\&R is still a very attractive choice for policy-makers who need to address urban traffic congestion issues. Improved social welfare can be achieved through careful and appropriate planning and design.

In further research, we will consider the heterogeneity of the commuter population with respect to different income levels, and will analyze the effects of some of the P\&R mode's attributes, e.g., in-vehicle congestion, metro fare, etc.

\section{Acknowledgements}

This research was supported by the National Natural Science Foundations of China (Nos. 51178403 and 51108391). 


\section{References}

[1] I.D.M. Bos, R.V.D. Heijden, E.J.E. Molin, et al., The choice of park and ride facilities: an analysis using a context-dependent hierarchical choice experiment, Environment and Planning A, 2004, 36(9): 1673-1686.

[2] R. Spillar, Park and Ride Planning and Design Guidelines, New York: Parsons Brinckerhoff Inc., 1997.

[3] H.Z. Guan, K. Nishii, A modeling method for estimating the park and bus ride demand, In: The 2nd International Conference on Traffic and Transportation Studies, Beijing, Reston, Virginia: ASCE, 2000: 378-383.

[4] W.H.K. Lam, M.H. Nicholas, H.P. Lo, How park-and-ride schemes can be successful in eastern Asia, Journal of Urban Planning and Development, 2001, 127(2): 63-78.

[5] Z.C. Li, W.H.K. Lam, S.C. Wong, et al., Modeling parkand-ride services in a multimodal transport network with elastic demand, Transportation Research Record, 2007, 1994: 101-109.

[6] S. Clark, D. Watling, Modeling network travel time reliability under stochastic demand, Transportation Research B, 2005, 39(2): 119-140.

[7] Y.F. Yin, W.H.K. Lam, M.A. Miller, Simulation-based reliability assessment approach for congested transit network, Journal of Advanced Transportation, 2003, 38(1): 27-44.

[8] Z.C. Li, W.H.K. Lam, S.C. Wong, et al., Reliability evaluation for stochastic and time-dependent networks with multiple parking facilities, Network and Spatial Economics, 2008, 8(4): 355-381.

[9] M.A.P. Taylor, Travel time variability: the case of two public modes, Transportation Science, 1982, 16(4): 517-521.

[10] V.R. Vuchic, Urban Transit: Operation, Planning and Economics, Hoboken: John Wiley \& Sons, Inc., 2005.

[11] R.B. Noland, K.A. Small, P.M. Koskenoja, et al., Simulating travel reliability, Regional Science and Urban Economics, 1998, 28(5): 535-564.

[12] W.B. Fan, D.M. Yang, Modeling park-and-ride behavior in a stochastic transportation network with capacity constraints, In: The 2nd International Conference of Transportation Engineering, Chengdu, Reston: ASCE, 2009.

[13] K.A. Small, Urban Transportation Economics, Switzerland: Harwood Academic Publishers, 1992.

[14] B. Ran, D. Boyce, Modeling Dynamic Transportation Networks: An Intelligent Transportation System Oriented Approach, Berlin: Springer, 1996.

[15] W.H.K. Lam, Z.C. Li, H.J. Huang, et al., Modeling timedependent travel choice problems in road networks with multiple user classes and multiple parking facilities, Transportation Research Part B, 2006, 40(5): 368-395.

[16] Z.C. Li, H.J. Huang, Fixed-point model and schedule reliability of morning commuting in stochastic and timedependent transport networks, Lecture Notes in Computer Science, 2005, 3828: 777-787.

(Editor: Yao ZHOU) 